12,18

\title{
Кластерная модель латеральной графеноподобной гетероструктуры: оценки перехода заряда
}

\author{
() С.Ю. Давыдов
}

Физико-технический институт им. А.Ф. Иофрфе РАН,

Санкт-Петербург, Россия

E-mail: Sergei_Davydov@mail.ru

(Поступила в Редакцию 28 фревраля 2018 г.)

Для описания зигзаго- и креслообразного контактов адсорбированных на металлической подложке графена и гексагонального двумерного бинарного соединения предложена простая кластерная модель. Подробно рассмотрена гетероструктура (ГС) графен-нитрид бора. Получены аналитические выражения для локальных плотностей состояний и чисел заполнения приконтактных атомов. Подробно проанализирован переход заряда для квазисвободных ГС. Приведены оценки энергии связи ГС с металлической подложкой.

DOI: 10.21883/FTT.2018.09.46404.045

\section{1. Введение}

Интерес к изучению латеральных (плоскостных) графеноподобных гетероструктур (ГС) продиктован, в первую очередь, потребностями наноэлектроники. В этом плане в настоящее время наиболее популярны структуры, образованные однолистным графеном (Gr) и гексагональным нитридом бора h-BN, чьи постоянные решетки совпадают с точностью $\sim 2 \%$ (см. работы [1-7] и ссылки, приведенные там). Отметим, что латеральная система Gr-h-BN уникальна: в одной кристаллографической двумерной структуре непрерывным образом (без каких-либо значимых нарушений симметрии) сочетаются полуметалл и диэлектрик с широкой $(\sim 6 \mathrm{eV})$ запрещенной зоной.

В работе [8] нами были предложены простые схемы теоретического рассмотрения свободных латеральных ГС, среди которых, в частности, была предложена модель взаимодействующих димеров, позволяющая простейшим образом оценить переход заряда (один из главных, по-видимому, эффектов в подобного рода гетероконтактах). В работе [9] для графеноподобных соединений была использована кластерная модель. Здесь, с учетом результатов $[8,9]$, мы рассмотрим ГC $\mathrm{Gr}-\mathrm{h}-\mathrm{BN}$ как в свободном состоянии, так и сформированную на металле. При этом мы будем считать для простоты, что расстояния между ближайшими соседями (б.с.) контактирующих двумерных (2D) полубесконечных решеток одинаковы. Отметим, что за исключением последнего упрощения (т. е. игнорирования слабого рассогласования решеток, что ведет, в принципе, к появлению дислокаций несоответствия), предлагаемая модель может быть использована и для других гетеропереходов, образованных графеноподобными соединениями.

\section{2. Кластерные модели гетеропереходов: общие соотношения}

2.1. Контакт зигзагообразного типа.На рис. 1 изображены кластеры, моделирующие ГС, образованные контактом одноатомной и двухатомной полубесконечных гексагональных двумерных решеток. Рассмотрим сначала случай контакта зигзагообразных краев таких решеток (рис. 1,a), моделируя такой контакт кластером, включающим атомы 1-6. Будем считать, что этот кластер расположен на подложке, так что составляющие его атомы являются на самом деле адатомами. В рамках такого „адсорбционного подхода“ $[10,11]$, функции Грина $G_{i j}$ (индексы $i$ и $j$ нумеруют адатомы кластера) могут быть найдены с помощью уравнений Дайсона $G_{i j}=g_{i i}+g_{i i} T_{i l} G_{i j}$, где $g_{i i}-$ диагональная функция Грина невзаимодействующих адатомов $\left(g_{i j}=g_{i i} \delta_{i j}\right.$, где $\delta_{i j}$ - символ Кронекера) и подразумевается суммирование по повторяющимся индексам. Будем считать, что ниже линии стыка, изображенной на рис. 1, $a$ штриховой линией, находится графен, а выше - гексагональный нитрид бора. Когда в контакт вступают адатом углерода и адатом нитрида бора $A(B)$, тогда, как показано в п. 1 Приложения, функции Грина для приконтактных атомов имеют вид

$$
\begin{gathered}
G_{11}^{A(B)}=g\left(1-2 g^{2} t^{2}-\frac{g g_{A(B)} \tilde{t}^{2}}{1-2 g_{A} g_{B} t^{2}}\right)^{-1}, \\
G_{22}^{A(B)}=g_{A(B)}\left(1-2 g_{A} g_{B} t^{2}-\frac{g g_{A(B)} \tilde{t}^{2}}{1-2 g^{2} t^{2}}\right)^{-1} .
\end{gathered}
$$

Определение входящих в (1) и (2) величин приведено в Приложении, п. 1.

Положим функции Грина невзаимодействующих адатомов равными $g^{-1}=\omega-\varepsilon-\Lambda(\omega)+i \Gamma(\omega), g_{A(B)}^{-1}=$ $=\omega-\varepsilon_{A(B)}-\Lambda_{A(B)}(\omega)+i \Gamma_{A(B)}(\omega)$, где $\varepsilon\left(\varepsilon_{A(B)}\right), \Lambda(\omega)$ 


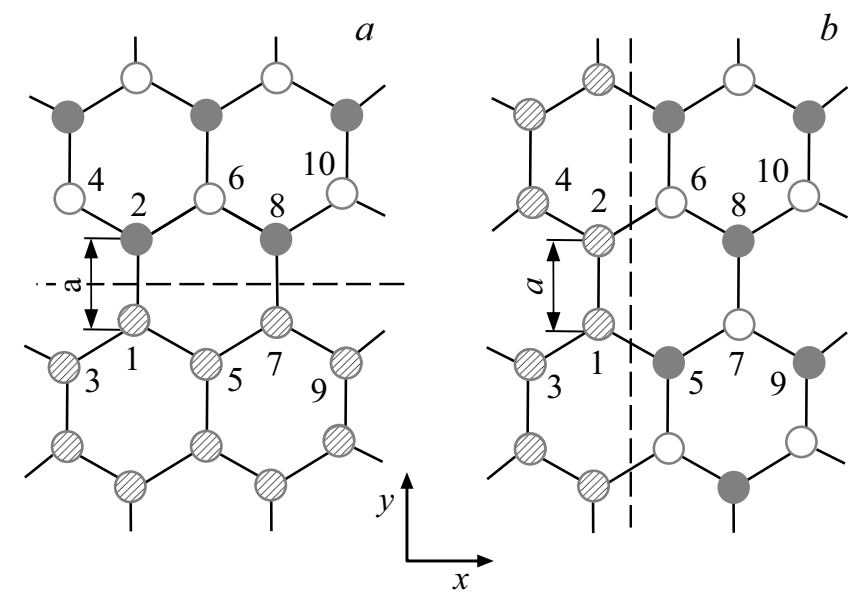

Рис. 1. Кластеры, моделирующие гетероконтакт $(a)$ зигзагообразного и $(b)$ креслообразного типов. Заштрихованными кружками обозначены атомы углерода графена, светлыми и темными кружками - атомы гексагонального нитрида бора, $a-$ расстояние между ближайшими соседями.

$\left(\Lambda_{A(B)}(\omega)\right)$ и $\Gamma(\omega)\left(\Gamma_{A(B)}(\omega)\right)$ есть энергия $p_{z}$-орбитали, функция сдвига и функция уширения атома графена (атомов нитрида бора) - см. подробнее п. 3 далее, $\omega-$ энергетическая переменная $[10,11]$. Отметим, что при $\tilde{t}=0$ функции Грина (1) и (2) переходят в функцию Грина центрального атома адсорбированного тримера $G_{2}^{\text {trim }}$ (см. формулу (8) в [12]). Для случая, когда адатом графена взаимодействует с адатомом нитрида бора $B$, функции Грина $G_{11}^{B}$ и $G_{22}^{B}$ даются формулами (1) и (2) с заменой $g_{A}$ на $g_{B}$.

Энергетические уровни ГС, определяемые полюсами функций Грина (1) и (2), находятся из уравнений

$$
\begin{aligned}
& \operatorname{Re}\left[\left(1-2 g^{2} t^{2}\right)\left(1-2 g_{A} g_{B} t^{2}\right)-g g_{A} \tilde{t}^{2}\right]=0, \\
& \operatorname{Re}\left[\left(1-2 g^{2} t^{2}\right)\left(1-2 g_{A} g_{B} t^{2}\right)-g g_{B} \tilde{t}^{2}\right]=0 .
\end{aligned}
$$

Локальные плотности состояний на атомах 1 и 2 (в расчете на одно направление спина) равны $\rho_{11}^{A(B)}=-\pi^{-1} \operatorname{Im} G_{11}^{A(B)}, \rho_{22}^{A(B)}=-\pi^{-1} \operatorname{Im} G_{22}^{A(B)}$, а соответствующие числа заполнения при нулевой температуре есть

$$
n_{1(2)}^{A(B)}=2 \int_{-\infty}^{\varepsilon_{\mathrm{F}}} \rho_{11(22)}^{A(B)} d \omega,
$$

где $\varepsilon_{\mathrm{F}}$ - уровень Ферми системы и множитель 2 учитывает спин.

2.2. Контакт креслообразного типа.Для описания контакта, изображенного на рис. $1, b$, выберем два шестиатомных кластера: состоящие из атомов 1-6 для графена и 1, 2, 5-8 для BN (здесь учтена трансляционная симметрия кластеров вдоль линии контакта). Тогда можно показать (см. Приложение, п. 2), что интересующие нас функции Грина для адатомов графена и нитрида бора равны соответственно

$$
\begin{gathered}
G_{11(22)}^{A(B)}=g\left(1-g^{2} t^{2}-g g_{A(B)} \tilde{t}^{2}-\frac{g^{2} t^{2}}{1-g^{2} t^{2}-g g_{B(A)} \tilde{t}^{2}}\right)^{-1}, \\
G_{A(B)}=g_{A(B)}\left(1-g g_{A(B)} \tilde{t}^{2}-g_{A} g_{B} t^{2}\right. \\
\left.-\frac{g_{A} g_{B} t^{2}}{1-g g_{B(A)} \tilde{t}^{2}-g_{A} g_{B} t^{2}}\right)^{-1} \cdot
\end{gathered}
$$

Для функции Грина $G_{11(22)}^{A(B)}$ индексы указывают, что адатомы графена 1 и 2 связаны непосредственно с адатомами $A$ и $B$ нитрида бора.

Энергетические уровни ГС, определяемые полюсами функций Грина (5) и (6), находятся из уравнений

$$
\begin{gathered}
\operatorname{Re}\left[\left(1-g^{2} t^{2}-g g_{A} \tilde{t}^{2}\right)\left(1-g^{2} t^{2}-g g_{B} \tilde{t}^{2}\right)-g^{2} t^{2}\right]=0, \\
\operatorname{Re}\left[\left(1-g_{A} g_{B} t^{2}-g g_{A} \tilde{t}^{2}\right)\left(1-g_{A} g_{B} t^{2}-g g_{B} \tilde{t}^{2}\right)-g_{A} g_{B} t^{2}\right]=0 .
\end{gathered}
$$

Соответствующие плотности состояний и числа заполнения вычисляются так же как в п. 2.1.

\section{3. Свободная гетероструктура $\mathrm{Gr}-\mathrm{h}-\mathrm{BN}$}

Рассмотрим для начала свободные латеральные ГС, положив $V=V_{A(B)}=0$, так что функции Грина адатомов равны функциям Грина атомов $g=\Omega^{-1}$ и $g_{A(B)}=\Omega_{A(B)}^{-1}$, где $\Omega=\omega-\varepsilon+i s, \Omega_{A(B)}=\omega-\varepsilon_{A(B)}+i s, s=0^{+}-$ бесконечно малая константа с размерностью энергии (здесь и далее верхний индекс 0 относится к невзаимодействующим с подложкой структурам). Для дальнейшего анализа положим $\varepsilon=0$ и перейдем к относительным величинам $x=\omega / t, e_{A(B)}=\varepsilon_{A(B)} / t, \tau=\tilde{t} / t$.

Согласно таблицам атомных термов ХерманаСкиллмана [13], энергии $p$-состояний рассматриваемых атомов относительно вакуума в $\mathrm{eV}$ равна -6.64 (В), $-8.97(\mathrm{C}),-11.47(\mathrm{~N})$. В соответствии с теорией Харрисона [14] для межатомного расстояния $a=1.42 \AA$ энергия перехода $t=V_{p p o} \approx 2.38 \mathrm{eV}$, где $V_{p p \pi}$ - матричный элемент $\pi$-связи $p$-орбиталей. Тогда $e_{A} \approx 0.98(\mathrm{~B})$, $e_{B} \approx-1.05(\mathrm{~N})$. Для дальнейших оценок положим $e_{A}=-e_{B}=1$ и $\tau=1$.

В случае зигзагообразного контакта безразмерные уровни энергии, отвечающие уравнениям (3), равны $x_{1,5}=\mp 2.000, x_{2,6}= \pm 1.000, x_{3,7}=\mp 1.303, x_{4,8}= \pm 2.303$, где верхние знаки соответствуют первому из уравнений (3), нижние - второму. Отметим, что состояния $x_{k}<0$ являются связывающими, $x_{k}>0-$ антисвязывающими. Для нахождения чисел заполнения атомов воспользуемся схемой, описанной в п. 3 Приложения. Тогда для атомов углерода, спаренных непосредственно с атомами бора и азота, получаем соответственно числа заполнения $\bar{n}_{1}^{A}=\bar{n}_{\mathrm{C}}^{\mathrm{B}}=1.03$ и $\bar{n}_{1}^{\mathrm{B}}=\bar{n}_{\mathrm{C}}^{\mathrm{N}}=0.97$. Для атомов 
нитрида бора имеем $\bar{n}_{2}^{A}=\bar{n}_{\mathrm{B}}=0.48$ и $\bar{n}_{2}^{B}=\bar{n}_{\mathrm{N}}=1.52$. В случае креслообразного контакта согласно уравнениям (7) получаем следующие безразмерные уровни энергии: $x_{1,4}= \pm 2.119, x_{2,5}= \pm 0.407, x_{3,6}= \pm 1.159$ для графена и $x_{1,4}= \pm 2.250, x_{2,5}= \pm 0.329, x_{3,6}= \pm 1.352$ для $\mathrm{BN}$. При этом $\bar{n}_{1}^{A}=\bar{n}_{\mathrm{C}}^{\mathrm{B}}=1.08, \bar{n}_{2}^{B}=\bar{n}_{\mathrm{C}}^{\mathrm{N}}=0.92(\mathrm{Gr})$; $\bar{n}_{A}=\bar{n}_{\mathrm{B}}=0.60, \bar{n}_{\mathrm{B}}=\bar{n}_{\mathrm{N}}=1.40(\mathrm{BN})$.

\section{4. Гетероструктуры $\mathrm{Gr}-\mathrm{h}-\mathrm{BN}$ на металле}

Так как, насколько известно автору, в настоящее время латеральные структуры $\mathrm{Gr}-\mathrm{h}-\mathrm{BN}$ сформированы только на медной подложке [1-3], рассмотрим задачу о $Г C$ на металле. Применим так называемый адсорбционный подход, в рамках которого кластеры, изображенные на рис. 1, строятся не из свободных атомов, а из адатомов $[8,9]$.

Как известно $[8,9]$, полуширины квазиуровней адатомов графена и нитрида бора равны соответственно $\Gamma(\omega)=\pi V^{2} \rho_{\text {sub }}(\omega)$ и $\Gamma_{A(B)}(\omega)=\pi V_{A(B)}^{2} \rho_{\text {sub }}(\omega)$, где $V$ и $V_{A(B)}$ - матричные элементы взаимодействия адатом-подложка, $\rho_{\mathrm{sub}}(\omega)-$ плотность состояний подложки. Функции сдвига определяются как $\Lambda(\omega)=V^{2} P \int_{-\infty}^{\infty} d \omega^{\prime} \rho_{\text {sub }}\left(\omega^{\prime}\right)\left(\omega-\omega^{\prime}\right)^{-1}$ для адатомов графена и $\Lambda_{A(B)}(\omega)=V_{A(B)}^{2} P \int_{-\infty}^{\infty} d \omega^{\prime} \rho_{\text {sub }}\left(\omega^{\prime}\right)\left(\omega-\omega^{\prime}\right)^{-1}$ для адатомов В $(\mathrm{N})$, где $P-$ символ главного значения интеграла. Положив плотность состояний металлической подложки равной $\rho_{\text {sub }}(\omega)=\rho_{m}=$ const $[8,9]$, получим вместо функций $\Gamma(\omega)$ и $\Gamma_{A(B)}(\omega)$ не зависящие от энергии параметры $\Gamma_{m}=\pi V_{m}^{2} \rho_{m}$ и $\Gamma_{m A(B)}=\pi V_{m A(B)}^{2} \rho_{m}$. При этом функции сдвига $\Lambda_{m}(\omega)$ и $\Lambda_{m A(B)}(\omega)$ обращаются в нуль. Переходя к относительным единицам и вводя безразмерные параметры связи адатом-подложка $\gamma_{m}=\Gamma_{m} / t, \gamma_{m A(B)}=\Gamma_{m A(B)} / t$, получим приведенные (безразмерные) функции Грина $g^{*}=g t=\left(x+i \gamma_{m}\right)^{-1}$ и $g_{A(B)}^{*}=g_{A(B)} t=\left(x \mp 1+i \gamma_{A(B)}\right)^{-1}$ для адатомов графена и нитрида бора соответственно. Будем рассматривать плоскую ГС, считая, что листы графена и нитрида бора лежат в одной плоскости, т.е. находятся на одинаковом расстоянии от подложки (плоская ГС). Тогда, согласно теории Харрисона $[13,14], V_{m}=V_{m A(B)}$ и $\gamma_{m}=\gamma_{m A(B)} \equiv \gamma$.

Далее мы будем рассматривать квазисвободные латеральные ГС, полагая $\gamma^{2} \ll 1$. Этот предел (предел слабой связи ГС-подложка) представляется наиболее интересным, так как в случае сильной связи с металлической подложкой графеноподобные соединения во многом теряют свои уникальные черты $[15,16]$.

Можно показать, что в пределе малых $\gamma$ локальные плотности состояний могут быть представлены в виде суммы лоренцевых распределений с соответствующими весовыми множителями. Для зигзагообразного контакта, которому отвечают функции Грина (1) и (2) (в безразмерном виде - выражения (П4) и (П5)), приведенные локальные плотности состояний равны

$$
f_{1(2)}^{A(B)}(x)=\rho_{11(22)}^{A\{B\}} \cdot t=\sum_{k}\left(v_{11(22)}^{A(B)}\right)_{k} \rho\left(x-x_{k}\right),
$$

где $\rho\left(x-x_{k}\right)=\gamma / \pi\left[\left(x-x_{k}\right)^{2}+\gamma^{2}\right]$.

Для креслообразного контакта, описываемого функциями Грина (5) и (6) (или (П8) и (П9)), аналогичным образом получим

$$
\begin{gathered}
f_{1(2)}^{A(B)}(x)=\sum_{k} v_{k}^{A(B)} \rho\left(x-x_{k}\right), \\
f_{A(B)}(x)=\sum_{k}\left(v_{A(B)}\right)_{k} \rho\left(x-x_{k}\right) .
\end{gathered}
$$

В формулах (8)-(10) под $x_{k}$ понимаются полюса соответствующих функций Грина для свободной ГС, значения которых приведены в разделе 3 , весовые $v$-множители являются вычетами функций Грина свободных ГС (см. Приложение, п. 3). Таким образом, плотности состояний $(8)-(10)$ представляют собой суперпозицию лоренцевских распределений $\rho\left(x-x_{k}\right)$, отвечающих центрированным при $x=x_{k}$ квазиуровням с полушириной $\gamma$.

На рис. 2 и 3 приведены локальные плотности состо-

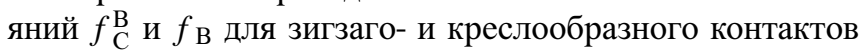
соответственно. Здесь функция $f_{\mathrm{C}}^{\mathrm{B}}$ отвечает адатому углерода графена, связанному с адатомом бора h-BN, функция $f_{\mathrm{B}}$ относится к адатому бора, взаимодействующему с адатомом углерода. Отметим, что имеют место равенства $f_{\mathrm{C}}^{\mathrm{B}}(x)=f_{\mathrm{C}}^{\mathrm{N}}(-x)$ и $f_{\mathrm{B}}(x)=f_{\mathrm{N}}(-x)$, вытекающие из значений полюсов $x_{k}$ и весовых $v$-множителей. Изначальная же причина такой симметрии связана с принятыми нами значениями атомных энергий $\varepsilon_{\mathrm{C}}=\varepsilon=0$ и $e_{\mathrm{B}}=-e_{\mathrm{N}}$. Стоит отметить, что приведенные на рис. 2 и 3 зависимости с качественной точки зрения близки к плотностям состояний, вычисленным из первых принципов $[4,5]$.

Для определения чисел заполнения адатомов при нулевой температуре следует проинтегрировать выражения (8)-(10) по $x$ от $-\infty$ (приближение бесконечно широкой зоны металлической подложки $[10,11])$ до приведенного уровня Ферми системы $e_{\mathrm{F}}=\varepsilon_{\mathrm{F}} / t$. Тогда для зигзагообразного контакта получим

$$
n_{1(2)}^{A(B)}=2 \sum_{k}\left(v_{11(22)}^{A(B)}\right)_{k} n_{k},
$$

а для креслообразного -

$$
n_{1(2)}^{A(B)}=2 \sum_{k} v_{k}^{A(B)} n_{k}, \quad n_{A(B)}=2 \sum_{k}\left(v_{A(B)}\right)_{k} n_{k},
$$

где $n_{k}=\pi^{-1} \operatorname{arctg}\left[\left(x_{k}-e_{\mathrm{F}}\right) / \gamma\right]$.

На рис. 4 и 5 представлены результаты расчета зависимостей чисел заполнения от безразмерной константы связи (приведенной полуширины квазиуровней) 
$\gamma$ для зигзаго- и креслообразного контактов. Полученные графики объясняются зависимостями значений $n_{k}$ от параметров $\gamma$ и $e_{\mathrm{F}}$. При $x_{k}>e_{\mathrm{F}}$ значения $n_{k}$ с ростом $\gamma$ увеличиваются, при $x_{k}<e_{\mathrm{F}}-$ убывают. Сдвиг $e_{\mathrm{F}}$ в сторону положительных энергий ведет к росту $n_{k}$.

Зависимость результирующих чисел заполнения от уровня Ферми легко понять из рассмотрения соответствующих локальных плотностей состояний (рис. 2 и 3): сдвигая уровень $e_{\mathrm{F}}$ вверх по шкале энергий $x$, мы увеличиваем числа заполнения. Зависимость эта носит ступенчатый характер: при прохождении $e_{\mathrm{F}}$ через
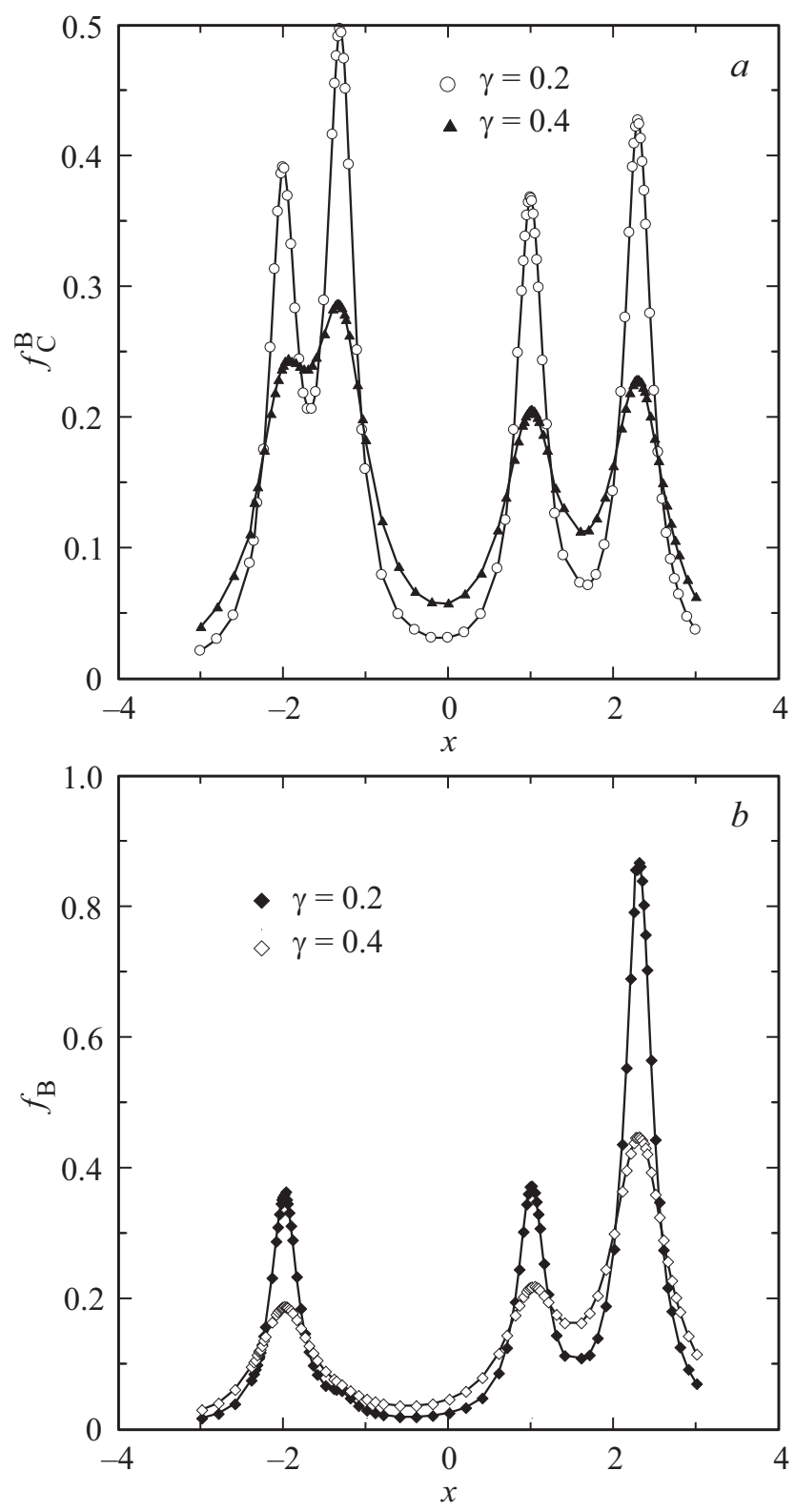

Pис. 2. Зигзагообразный контакт. Зависимости приведенных локальных плотностей состояний на $(a)$ адатоме углерода $f_{\mathrm{C}}^{\mathrm{B}}$, связанного с адатомом бора, и $(b)$ адатоме бора $f_{\text {в }}$ связанного с углеродом, от приведенной энергии $x$ для константы связи ГС-металл $\gamma=0.2$ (темные символы) и 0.4 (светлые символы).
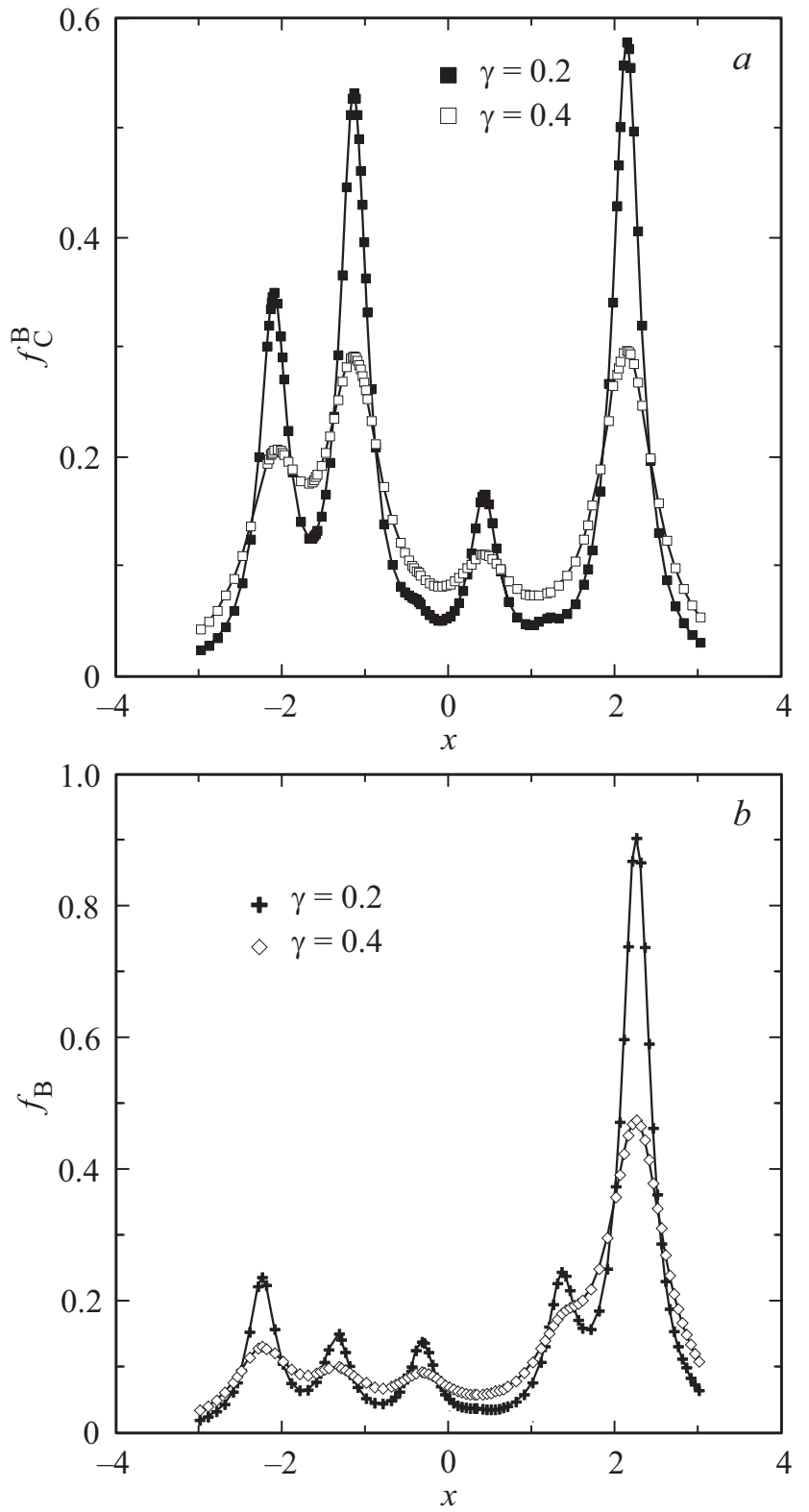

Рис. 3. Креслообразный контакт. То же, что на рис. 2.

максимумы плотности состояний имеет место быстрый рост числа заполнения, при прохождении минимумов рост чисел заполнения мал (близкий к плато). Такая ступенчатость тем резче, чем у́же квазиуровень. Действительно, воспользовавшись выражениями (9), (10), в пределе $\gamma \rightarrow 0$ получим

$$
\begin{gathered}
n_{1(2)}^{A(B)}=2 \sum_{k} v_{k}^{A(B)} \Theta\left(e_{\mathrm{F}}-x_{k}\right), \\
n_{A(B)}=2 \sum_{k}\left(v_{A(B)}\right)_{k} \Theta\left(e_{\mathrm{F}}-x_{k}\right),
\end{gathered}
$$

где $\Theta(y)-$ функция Хэвисайда, равная 1 при $y>0$, и 0 при $y<0$.

Заряды адатомов равны $Z_{\mathrm{C}}^{\mathrm{B}(\mathrm{N})}=1-n_{\mathrm{C}}^{\mathrm{B}(\mathrm{N})}$ и $Z_{\mathrm{B}(\mathrm{N})}=$ $=1-n_{\mathrm{B}(\mathrm{N})}$. Согласно расчетам для зигзагообразного 


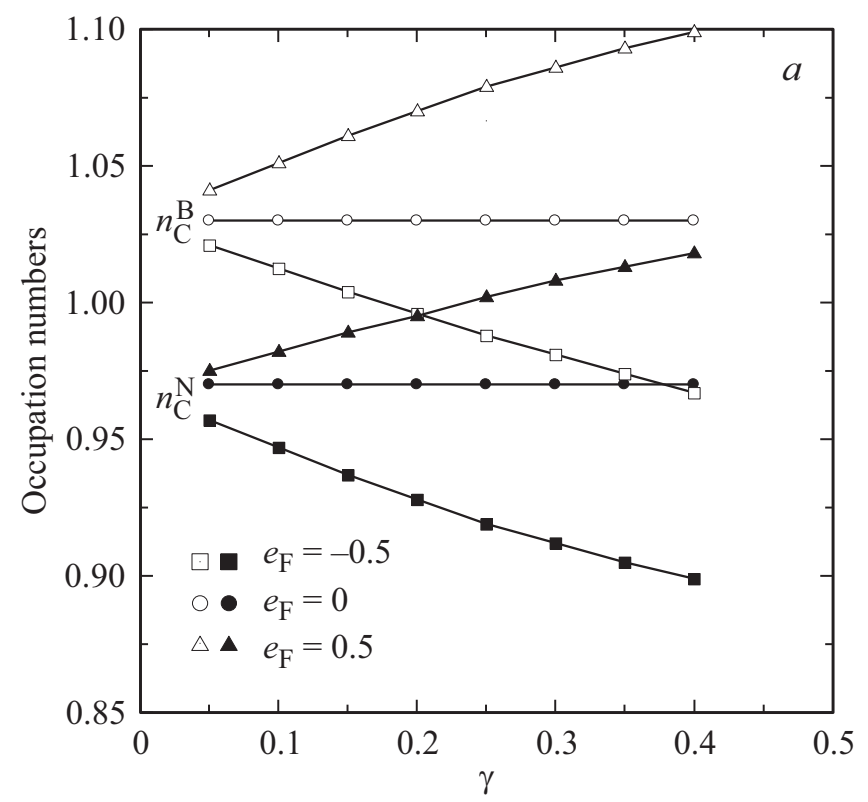

Заряды $Z_{\mathrm{B}}$ и $Z_{\mathrm{N}}$ уменьшается по величине, причем рост $\gamma$ приводит к их дополнительному уменьшению (рис. $4, b)$. Сдвиг $e_{\mathrm{F}}$ вверх к значению $e_{\mathrm{F}}=0.5$ вызывает уменьшение $Z_{\mathrm{C}}^{\mathrm{N}}$ вплоть до смены знака с положительного на отрицательный при $\gamma \approx 0.175$ и рост $\left|Z_{\mathrm{C}}^{\mathrm{B}}\right|$, усиливающийся с увеличением $\gamma$. Величины зарядов $Z_{\mathrm{B}}$ и $Z_{\mathrm{N}}$ при этом убывают, причем, эта убыль возрастает с увеличением $\gamma$.

Для креслообразного контакта при $e_{\mathrm{F}}=0$ заряды $Z_{\mathrm{C}}^{\mathrm{B}}$ и $Z_{\mathrm{C}}^{\mathrm{N}}$ малы (рис. $\left.5, a\right)$, близки по величине к зарядам свободной ГС, равным соответственно $\bar{Z}_{\mathrm{C}}^{\mathrm{B}}=-0.08$ и $\bar{Z}_{\mathrm{C}}^{\mathrm{N}}=+0.08$, и слабо убывают по величине с ростом $\gamma$. То же относится и к зарядам $Z_{\mathrm{B}}$ и $Z_{\mathrm{N}}$ (рис. $5, b$ ), равным для свободной ГС соответственно $\bar{Z}_{\mathrm{B}}=+0.40$ и $\bar{Z}_{\mathrm{N}}=-0.40$. Для $e_{\mathrm{F}}=-0.5$ имеем уменьшение как
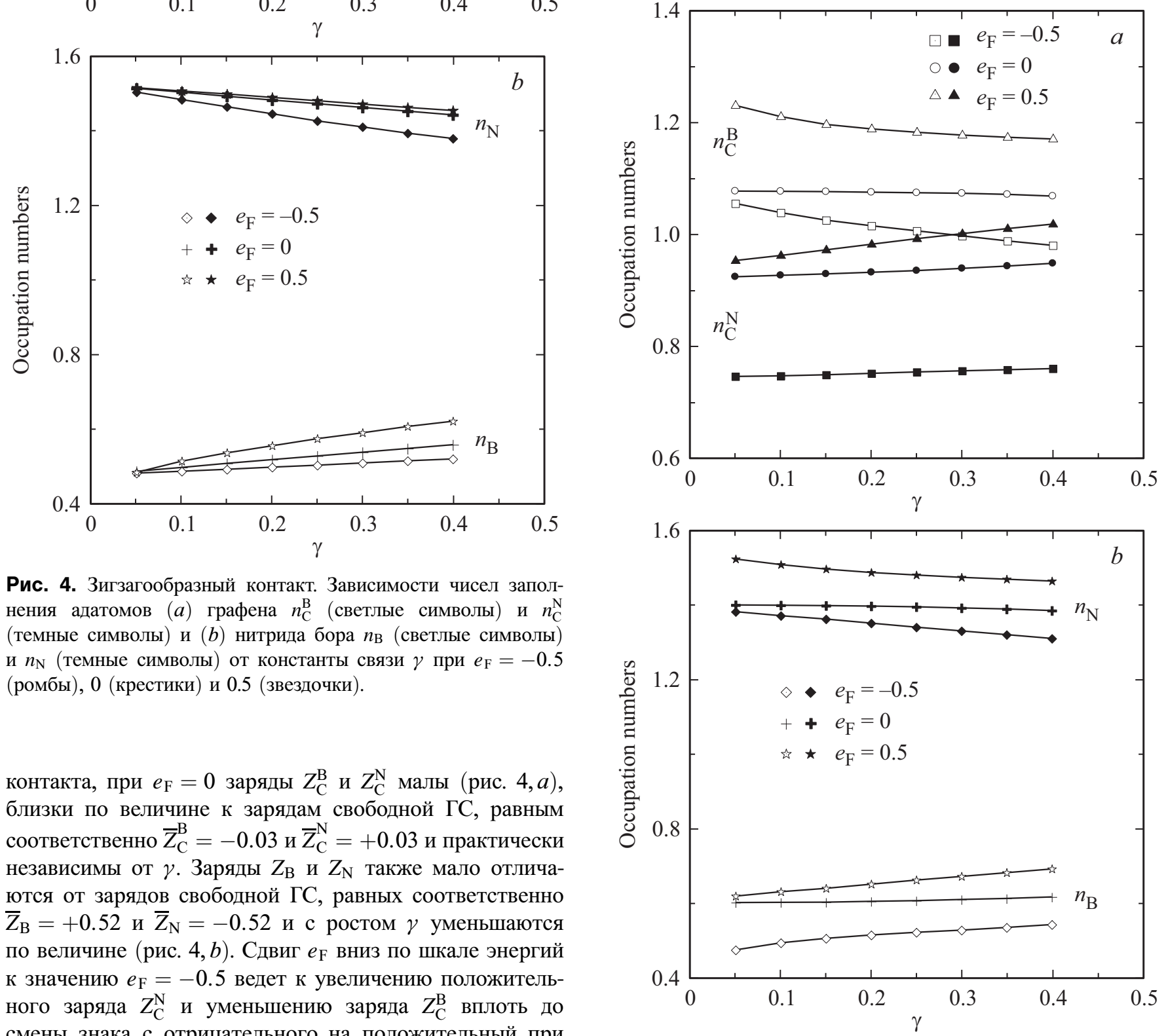

Рис. 4. Зигзагообразный контакт. Зависимости чисел заполнения адатомов $(a)$ графена $n_{\mathrm{C}}^{\mathrm{B}}$ (светлые символы) и $n_{\mathrm{C}}^{\mathrm{N}}$ (темные символы) и $(b)$ нитрида бора $n_{\text {В }}$ (светлые символы) и $n_{\mathrm{N}}$ (темные символы) от константы связи $\gamma$ при $e_{\mathrm{F}}=-0.5$ (ромбы), 0 (крестики) и 0.5 (звездочки).

контакта, при $e_{\mathrm{F}}=0$ заряды $Z_{\mathrm{C}}^{\mathrm{B}}$ и $Z_{\mathrm{C}}^{\mathrm{N}}$ малы (рис. $\left.4, a\right)$, близки по величине к зарядам свободной ГС, равным соответственно $\bar{Z}_{\mathrm{C}}^{\mathrm{B}}=-0.03$ и $\bar{Z}_{\mathrm{C}}^{\mathrm{N}}=+0.03$ и практически независимы от $\gamma$. Заряды $Z_{\mathrm{B}}$ и $Z_{\mathrm{N}}$ также мало отличаются от зарядов свободной ГС, равных соответственно $\bar{Z}_{\mathrm{B}}=+0.52$ и $\bar{Z}_{\mathrm{N}}=-0.52$ и с ростом $\gamma$ уменьшаются по величине (рис. $4, b)$. Сдвиг $e_{\mathrm{F}}$ вниз по шкале энергий к значению $e_{\mathrm{F}}=-0.5$ ведет к увеличению положительного заряда $Z_{\mathrm{C}}^{\mathrm{N}}$ и уменьшению заряда $Z_{\mathrm{C}}^{\mathrm{B}}$ вплоть до смены знака с отрицательного на положительный при $\gamma \approx 0.225$; с ростом $\gamma$ величина $Z_{\mathrm{C}}^{\mathrm{N}}$ возрастает (рис. $4, a$ ).

Рис. 5. Креслообразный контакт. То же, что на рис. 4. 


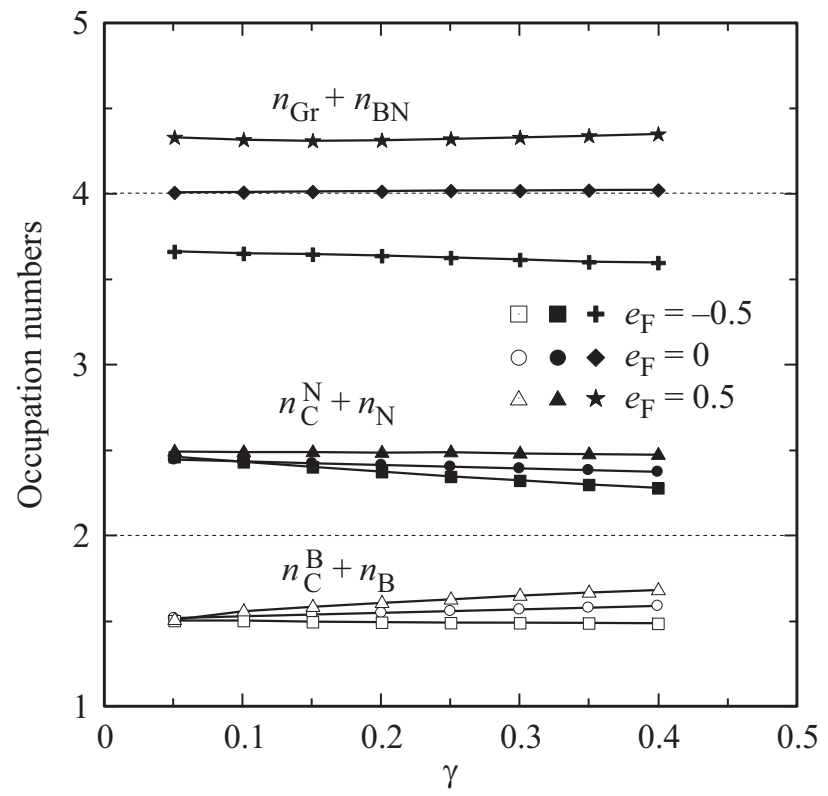

Рис. 6. Зависимости суммарного заполнения адатомов приконтактных областей для зигзагообразного $\left(n_{\mathrm{C}}^{\mathrm{B}}+n_{\mathrm{B}}\right.$ и $\left.n_{\mathrm{C}}^{\mathrm{N}}+n_{\mathrm{N}}\right)$ и креслообразного $\left(n_{\mathrm{Gr}}+n_{\mathrm{BN}}\right.$, где $n_{\mathrm{Gr}}=n_{\mathrm{C}}^{\mathrm{B}}+n_{\mathrm{C}}^{\mathrm{B}}$ и $\left.n_{\mathrm{BN}}=n_{\mathrm{B}}+n_{\mathrm{N}}\right)$ контактов от константы связи $\gamma$ при $e_{\mathrm{F}}=-0.5$ (квадраты и крестики), 0 (кружки и ромбы) и 0.5 (треугольники и звездочки). Штриховая и пунктирная линии отвечают заполнению атомов в свободной ГС.

величины заряда $Z_{\mathrm{C}}^{\mathrm{B}}$ вплоть до смены знака с отрицательного на положительный при $\gamma \approx 0.275$, так и заряда $Z_{\mathrm{C}}^{\mathrm{N}}$, для которого спад усиливается с ростом $\gamma$. Величины зарядов $Z_{\mathrm{B}}$ и $Z_{\mathrm{N}}$ также понижаются, причем это понижение пропорционально $\gamma$. Для $e_{\mathrm{F}}=0.5$ имеем уменьшение по величине заряда $Z_{\mathrm{C}}^{\mathrm{B}}$, усиливающееся с увеличением $\gamma$. Величина заряда $Z_{\mathrm{C}}^{\mathrm{N}}$ также убывает, меняя знак с положительного на отрицательный при $\gamma \approx 0.275$. Как при $e_{\mathrm{F}}=-0.5$, так и при $e_{\mathrm{F}}=0.5$ величины зарядов $Z_{\mathrm{B}}$ и $Z_{\mathrm{N}}$ также понижаются, причем это понижение пропорционально $\gamma$.

Представляет интерес суммарное заполнение контактирующих адатомов графена и нитрида бора. На рис. 6 для зигзагообразного контакта приведены значения заполнения двух атомов $n_{\mathrm{C}}^{\mathrm{B}}+n_{\mathrm{B}}$ и $n_{\mathrm{C}}^{\mathrm{N}}+n_{\mathrm{N}}$, образующих элементарную ячейку, которую можно транслировать вдоль линии контакта. Пунктирная линия отвечает соответствующему суммарному заполнению атомов свободной ГС, равному 2. Из рисунка следует, что на контакте графена с $\mathrm{N}$-краем нитрида бора сосредоточен отрицательный заряд $Z_{\mathrm{C}}^{\mathrm{N}}+Z_{\mathrm{N}} \approx-(0.3-0.5)$, тогда как на контакте с В-краем возникает положительный заряд $Z_{\mathrm{C}}^{\mathrm{B}}+Z_{\mathrm{B}}$ того же порядка. При этом положение уровня Ферми сказывается только на величине, но не на знаке заряда.

Для креслообразного контакта на рис. 6 представлена зависимость суммарного заполнения четырех атомов $n_{\mathrm{Gr}}+n_{\mathrm{BN}}$, где $n_{\mathrm{Gr}}=n_{\mathrm{C}}^{\mathrm{B}}+n_{\mathrm{C}}^{\mathrm{N}}$ и $n_{\mathrm{BN}}=n_{\mathrm{B}}+n_{\mathrm{N}}$, состав- ляющих элементарную ячейку, транслируемую вдоль контакта. При $e_{\mathrm{F}}=0$ значения $n_{\mathrm{Gr}}+n_{\mathrm{BN}}$ практически совпадают с заполнением атомов свободной ГС, равным 4 и обозначенном на рисунке штриховой линией. При $e_{\mathrm{F}}=-0.5$ на четырех атомах приконтактной области локализован положительный заряд $Z_{\mathrm{Gr}}+Z_{\mathrm{BN}} \approx 0.4$, при $e_{\mathrm{F}}=0.5-$ отрицательный заряд $Z_{\mathrm{Gr}}+Z_{\mathrm{BN}} \approx-0.3$. Отметим весьма слабую зависимость суммарного заряда от параметра $\gamma$.

Приведем теперь простые полуколичественные оценки энергии адсорбционной связи $E_{\text {ads }}$ структуры $\mathrm{Gr}-\mathrm{h}-\mathrm{BN}$ с металлической подложкой. Как известно $[9,10]$, оценить эту энергию можно, представив ее в виде $E_{\text {ads }}=E_{\text {met }}+E_{\text {ion, }}$, где $E_{\text {met }}$ и $E_{\text {ion }}$ есть металлическая и ионная составляющие энергии связи. Порядок той части величины энергии $E_{\text {met }}$, которая связана с переходом заряда между ГС и подложкой, можно оценить, исходя из соотношения неопределенностей $[10,11]$. Пусть при помещении адатома на поверхность металла $\Delta Z_{a}>0$ электронов перешло с адатома в металлическую подложку. При этом имеет место делокализация электронов, т.е. переход электрона из локализованного состояния ГС с радиусом локализации $\sim a=1.42 \AA$ в полностью делокализованное металлическое состояние. При этом на один адатом получаем выигрыш в кинетической энергии $\Delta E_{\text {kin }} \sim-\Delta Z_{a} \hbar^{2} / 2 m a^{2}$, где $\hbar-$ приведенная постоянная Планка, $m-$ масса свободного электрона. Обратный эффект имеет место, когда $\Delta Z_{a}<0$, что отвечает переходу электронов из делокализованного металлического состояния на ГС, сопровождаемого проигрышем в кинетической энергии $E_{\text {met }}=\Delta E_{\text {kin }} \sim-\Delta Z_{a} \hbar^{2} / 2 m a^{2}$ (в ед. $\mathrm{eV}$ ). Учитывая, что множитель $\hbar^{2} / 2 m a^{2} \sim 2 \mathrm{eV}$, можем записать металлическую составляющую энергии связи, приходящуюся на элементарную ячейку, в виде

$$
\begin{aligned}
& E_{\text {met }}^{\text {zigzag }}(\mathrm{C}-\mathrm{B}) \sim-2\left(2-n_{\mathrm{C}}^{\mathrm{B}}-n_{\mathrm{B}}\right), \\
& E_{\text {met }}^{\text {zigzag }}(\mathrm{C}-\mathrm{N}) \sim-2\left(2-n_{\mathrm{C}}^{\mathrm{N}}-n_{\mathrm{N}}\right)
\end{aligned}
$$

для зигзагообразных контактов графена с B- и N-краями нитрида бора соответственно и

$$
E_{\mathrm{met}}^{\text {chair }} \sim-2\left(4-n_{\mathrm{Gr}}-n_{\mathrm{BN}}\right)
$$

для креслообразного контакта, где все энергии выражены в $\mathrm{eV}$. Знак, порядок величины и характер изменения энергий (15) и (16) с ростом легко определить, воспользовавшись графиками рис. 6.

Ионную составляющую $E_{\text {iоn }}$ для одного адатома с зарядом $Z_{a}$ можно оценить классическим электростатическим выражением $E_{\text {ion }}=-Z_{a}^{2} e^{2} / 4 d$, где $d-$ длина адсорбционной связи (см. подробнее $[10,11])$, а в качестве заряда адатома $Z_{a}$ выступают $Z_{\mathrm{C}}^{\mathrm{B}}, Z_{\mathrm{C}}^{\mathrm{N}}, Z_{\mathrm{B}}$ или $Z_{\mathrm{N}}$. Полагая $d \sim 3-4 \AA$ [17], что характерно для квазисвободной эпитаксиальной структуры, в ед. $\mathrm{eV}$ получим $E_{\mathrm{ion}} \sim-Z_{a}^{2}$ на один адатом. В расчете на элементарную ячейку в случае зигзагообразного контакта имеем энергию 
ионной составляющей $E_{\text {ion }}^{\text {zigzag }}(\mathrm{C}-\mathrm{B}) \sim-\left[\left(Z_{\mathrm{C}}^{\mathrm{B}}\right)^{2}+Z_{\mathrm{B}}^{2}\right]$ и $E_{\text {ion }}^{\text {zigzag }}(\mathrm{C}-\mathrm{N}) \sim-\left[\left(Z_{\mathrm{C}}^{\mathrm{N}}\right)^{2}+Z_{\mathrm{N}}^{2}\right]$ для контакта графена с B- и $\mathrm{N}$-краями нитрида бора соответственно. При креслообразном контакте имеем $E_{\text {ion }}^{\text {chair }} \sim-\left[\left(Z_{\mathrm{C}}^{\mathrm{B}}\right)^{2}+\left(Z_{\mathrm{C}}^{\mathrm{N}}\right)^{2}\right.$ $\left.+Z_{\mathrm{B}}^{2}+Z_{\mathrm{N}}^{2}\right]$. Воспользовавшись для качественных оценок значениями зарядов для свободной ГС, получим для обоих типов зигзагообразных контактов $E_{\text {ion }}^{\text {zigzag }} \sim-0.5 \mathrm{eV}$ и $E_{\text {ion }}^{\text {chair }} \sim-0.3 \mathrm{eV}$.

\section{5. Обсуждение результатов и заключительные замечания}

В настоящей работе мы считали эпитаксиальную структуру $\mathrm{Gr}-\mathrm{h}-\mathrm{BN}$ на металле плоской. Чтобы продемонстрировать справедливость этого предположения, рассмотрим энергию связи графена и нитрида бора. Воспользовавшись методом Харрисона $[14,18]$, легко показать (пренебрегая вкладом металличности связи), что энергия двухэлектронной связи $E_{b}=-2 V_{2}\left(1-2 \alpha_{c}^{2}\right) / \alpha_{c}$, где $V_{2}=12.32 \mathrm{eV}$ - ковалентная энергия $\sigma$-связи $s p^{2}$-орбиталей для межатомного расстояния $a=1.42 \AA ; \alpha_{c}=V_{3} /\left(V_{2}^{2}+V_{3}^{2}\right)^{1 / 2}-$ ковалентность связи, $V_{3}$ —полярная энергия, равная половине модуля разности энергий, участвующих в связи $s p^{2}$-орбиталей $\varepsilon_{h}=\left(\varepsilon_{s}+2 \varepsilon_{p}\right) / 3, \quad \varepsilon_{s}$ и $\varepsilon_{p}-$ энергии $s$ - и $p$-орбиталей $[13,14]$. Воспользовавшись таблицами атомных термов Хермана-Скиллмана [13], получим $E_{b}(\mathrm{C}-\mathrm{B}) \approx E_{b}(\mathrm{C}-\mathrm{N}) \approx-8.63 \mathrm{eV}$ для связей атомов углерода графена с атомами бора и азота нитрида бора. Отметим, что энергия связи графена равна $E_{b}(\mathrm{C}-\mathrm{C}) \approx-8.21 \mathrm{eV}$, для нитрида бора получаем $E_{b}(\mathrm{~B}-\mathrm{N}) \approx-9.90 \mathrm{eV}$. Далее, в соответствии с моделью Фриделя [19] положим плотность состояний для $d$-зоны $\rho_{m}=10 / W_{m}$, где $W_{m}=2.8 \mathrm{eV}$. Так как $\gamma=\pi \rho_{m} V_{m}^{2} / t$, получим $V_{m} \approx 0.21 \mathrm{eV}$ для $\gamma=0.2$ и $V_{m} \approx 0.29 \mathrm{eV}$ для $\gamma=0.4$. Так как $V_{m} \ll V_{2}$ и $\left|E_{\text {ion }}^{\text {zigzag }}\right| \ll V_{2},\left|E_{\text {ion }}^{\text {chair }}\right| \ll V_{2}$, предположение о плоской ГС справедливо. Если, однако, рассматривать ковалентную связь ГС с подложкой (см., например, [20]), то возможна гофрировка ГС, отмеченная в работе [4].

Из рассмотрения плотностей состояний (рис. 2, 3) следует, что энергетические щели отсутствуют как для зигзагообразного, так и для креслообразного контактов, т.е. система является металлической. Можно лишь говорить о щелях подвижности (аналогично аморфным полупроводникам), соответствующих минимумам плотностей состояний. Наш вывод о металличности эпитаксиальной ГС совпадает с выводом работы [4].

Для анализа полученных результатов по переходу заряда рассмотрим димеры $\mathrm{C}_{2}$ и $\mathrm{BN}$. Легко показать (см., например, [11-13]), что отвечающие этим молекулам плотности состояний (на атомную $p_{z}$-орбиталь) имеют вид $\rho_{\mathrm{C}}=\delta(x-1)+\delta(x+1)$ и $\rho_{\mathrm{BN}}=(1-1 / \sqrt{2}) \delta(x-\sqrt{2})+(1+1 / \sqrt{2}) \delta(x+\sqrt{2})$, так что числа заполнения $p$-состояний равны 1 для атомов $\mathrm{C}$, 0.29 для атома В и 1.71 для атома N. Сравнивая эти значения с числами заполнения $\bar{n}_{\mathrm{C}}^{\mathrm{B}}=1.03, \bar{n}_{\mathrm{C}}^{\mathrm{N}}=0.97$ для зигзагообразного контакта и $\bar{n}_{\mathrm{C}}^{\mathrm{B}}=1.08, \bar{n}_{\mathrm{C}}^{\mathrm{N}}=0.92$, следует сделать вывод, что переход заряда для атомов углерода в свободной кластерной ГС чрезвычайно мал по сравнению с углеродным димером. С другой стороны, для атомов бора и азота в свободной кластерной ГС числа заполнения $\bar{n}_{\mathrm{B}}=0.48, \bar{n}_{\mathrm{N}}=1.52$ для зигзагообразного контакта и $\bar{n}_{\mathrm{B}}=0.60, \bar{n}_{\mathrm{N}}=1.40$ для креслообразного контакта, что существенно отличается от заполнения атомов димера BN.

Еще раз следует подчеркнуть, что материал, изложенный в п. 2, применим для моделирования любого гексагонального бинарного 2D-компонента ГC, у которого расстояние между б. с. не слишком сильно отличается от соответствующего значения для графена, что позволяет ввести единую референтную решетку. Отметим также, что возможны и другие, более сложные структурные варианты контактов в системе Gr-h-BN [4-6].

\section{Приложение}

1. Для элемента структуры, изображенного на pис. $1, a$, все матричные элементы взаимодействия ближайших соседей $T_{i j}$ равны $t$. Для большей общности положим, однако, для связей атомов 1 и 7 с атомами 2 и 8 матричные элементы $T_{i j}\left|T_{12}\right|=\left|T_{78}\right|=\tilde{t}$. Тогда, включив в модельный кластер только атомы 1-6, имеем следующую цепочку уравнений Дайсона: $G_{11}=g_{11}+g_{11} t\left(G_{31}+G_{51}\right)+g_{11} \tilde{t} G_{21}, \quad G_{31}=g_{33} t G_{11}$, $G_{51}=g_{55} t G_{11}, \quad G_{21}\left[1-g_{22}\left(g_{44}+g_{66}\right) t^{2}\right]=g_{22} \tilde{t} G_{11}$. Отсюда

$$
G_{11}=g_{11}\left(1-g_{11}\left(g_{33}+g_{55}\right) t^{2}-\frac{g_{11} g_{22} \tilde{t}^{2}}{1-g_{22}\left(g_{44}+g_{66}\right) t^{2}}\right)^{-1} .
$$

Из соображений симметрии можем записать $g_{11}=g_{33}=g_{55}=g_{C} \equiv g, \quad g_{22}=g_{88} \equiv g_{A}, \quad g_{44}=g_{66}=$ $=g_{1010} \equiv g_{B}$. Выражение для функции Грина $G_{22}$ легко получить из (П1) путем следующей замены индексов функций $g_{i i}: \quad 11 \rightarrow 22, \quad 33 \rightarrow 44,55 \rightarrow 66$. С учетом этих соотношений выражение (П1) переходит в выражения (1) и (2).

2. Для шестиатомных кластеров 1-6 (графен) и 1 , $2,5-8$ (нитрид бора), изображенных на рис. $1, b, \mathrm{c}$ помощью уравнения Дайсона получим для адатома 1 графена, непосредственно связанного с адатомом 5 (типа $A$ ) нитрида бора, функцию Грина вида

$$
\begin{aligned}
G_{11}^{A}=g_{11}\left(1-g_{11} g_{55} \tilde{t}^{2}-g_{11} g_{33} t^{2}\right. & \\
& \left.-\frac{g_{11} g_{22} t^{2}}{1-g_{22} g_{44} t^{2}-g_{22} g_{66} \tilde{t}^{2}}\right)^{-1} .
\end{aligned}
$$

Функцию Грина $G_{22}^{B}$ для адатома графена 2 легко получить, заменяя индексы 1 на 2, 5 на 6 и 3 на 4. Для 
адатома 5 (типа $A$ ) нитрида бора функция Грина имеет вид

$$
\begin{aligned}
G_{55}=g_{55}\left(1-g_{55} g_{11} \tilde{t}^{2}-g_{55} g_{77} t^{2}\right. \\
\\
\left.\quad-\frac{g_{66} g_{55} t^{2}}{1-g_{66} g_{88} t^{2}-g_{66} g_{22} \tilde{t}^{2}}\right)^{-1} .
\end{aligned}
$$

Функция Грина для адатома 6 (тип $B$ ) получается из (П3) путем описанных выше замен с дополнительными заменами 7 на 3 и 8 на 4. Учитывая, что $g_{11}=g_{22}=$ $=g_{33}=g_{44}=g, g_{55}=g_{A}, g_{66}=g_{B}$, получим формулы (6) и (7).

3. Перепишем функции Грина (1) и (2), отвечающие зигзагообразному контакту, в безразмерном виде

$$
\begin{aligned}
& \left(G_{11}^{A(B)}\right)^{*}=G_{11}^{A(B)} t=\frac{x\left(x^{2}-3\right)}{\prod_{k}\left(x-x_{k}+i s^{\prime}\right)}, \\
& \left(G_{22}^{A(B)}\right)^{*}=G_{22}^{A(B)} t=\frac{(x \pm 1)\left(x^{2}-2\right)}{\prod_{k}\left(x-x_{k}+i s^{\prime}\right)},
\end{aligned}
$$

где $x_{k}$ - безразмерные корни уравнения (5), значения которых приведены в основном тексте статьи для случая $\tau=1, s^{\prime}=0^{+}-$бесконечно малая безразмерная константа.

Для свободной ГС соответствующие приведенные плотности состояний равны

$$
\left(\rho_{11(22)}^{A(B)}\right)^{*}=\rho_{11}^{A(B)} t=\sum_{k}\left(v_{11(22)}^{A(B)}\right)_{k} \delta\left(x-x_{k}\right)
$$

где $\left(v_{i(2)}^{a(B)}\right)_{k}$ - числа заполнения состояния $k$, равные вычетам функций (П4) и (П5) в точке $x_{k}$, причем $\sum_{k}\left(v_{1(2)}^{A(B)}\right)_{k}=1$. В соответствии с (4), числа заполнения атомов равны

$$
n_{1(2)}^{A(B)}=2 \sum_{\text {occ. }} v_{11(22)}^{A(B)}
$$

где аббревиатура осс. означает суммирование по занятым состояниям.

Функции Грина (5) и (6), отвечающие креслообразному контакту, в безразмерном виде есть:

$$
\begin{aligned}
& \left(G_{11}^{A}\right)^{*}=\frac{x(x-1)\left(x^{3}+x^{2}-2 x-1\right)}{\prod_{k}\left(x-x_{k}+i s^{\prime}\right)}, \\
& \left(G_{11}^{B}\right)^{*}=\frac{x(x+1)\left(x^{3}-x^{2}-2 x+1\right)}{\prod_{k}\left(x-x_{k}+i s^{\prime}\right)}
\end{aligned}
$$

для атомов графена, контактирующих с атомами $A$ и $B$ нитрида бора, и

$$
\begin{aligned}
& \left(G_{A}\right)^{*}=\frac{x(x+1)\left(x^{3}-3 x+1\right)}{\prod_{k}\left(x-x_{k}+i s^{\prime}\right)}, \\
& \left(G_{B}\right)^{*}=\frac{x(x-1)\left(x^{3}-3 x-1\right)}{\prod_{k}\left(x-x_{k}+i s^{\prime}\right)}
\end{aligned}
$$

для атомов $A$ и $B$ нитрида бора. Соответствующие приведенные плотности состояний и числа заполнения даются формулами, аналогичными (П6) и (П7).

Приведем значения весовых множителей $\left(v_{i(2)}^{a(B)}\right)_{k}$ для всех рассматриваемых нами ГС. В случае зигзагообразного контакта имеем для атомов углерода графена: $\left(v_{11}^{A}\right)_{k_{1}}=0.222,\left(v_{11}^{A}\right)_{k_{2}}=0.222,\left(v_{11}^{A}\right)_{k_{3}}=0.293$, $\left(v_{11}^{A}\right)_{k_{4}}=0.262$; то же для атомов $B$. Для атомов нитрида бора: $\left(v_{22}^{A}\right)_{k_{1}}=0.222,\left(v_{22}^{A}\right)_{k_{2}}=0.222,\left(v_{22}^{A}\right)_{k_{3}}=0.016$, $\left(v_{22}^{A}\right)_{k_{4}}=0.540$; то же для атомов $B$.

В случае креслообразного контакта имеем для приконтактных атомов углерода графена: $\left(v_{11}^{A}\right)_{k_{1}}=0.360$, $\left(v_{11}^{A}\right)_{k_{2}}=0.092,\left(v_{11}^{A}\right)_{k_{3}}=0.009,\left(v_{11}^{A}\right)_{k_{4}}=0.205,\left(v_{11}^{A}\right)_{k_{5}}=$ $=0.012, \quad\left(v_{11}^{A}\right)_{k_{6}}=0.322 ;\left(v_{11}^{B}\right)_{k_{1}}=0.205, \quad\left(v_{11}^{B}\right)_{k_{2}}=0.012$, $\left(v_{11}^{B}\right)_{k_{3}}=0.322,\left(v_{11}^{B}\right)_{k_{4}}=0.360,\left(v_{11}^{B}\right)_{k_{5}}=0.092,\left(v_{11}^{B}\right)_{k_{6}}=$ $=0.009$. Для атомов нитрида бора имеем: $\left(v_{A}\right)_{k_{1}}=0.572$, $\left(v_{A}\right)_{k_{2}}=0.004, \quad\left(v_{A}\right)_{k_{3}}=0.124, \quad\left(v_{A}\right)_{k_{4}}=0.142, \quad\left(v_{A}\right)_{k_{5}}=$ $=0.077, \quad\left(v_{A}\right)_{k_{6}}=0.082 ; \quad\left(v_{B}\right)_{k_{1}}=0.142, \quad\left(v_{B}\right)_{k_{2}}=0.077$, $\left(v_{B}\right)_{k_{3}}=0.082,\left(v_{B}\right)_{k_{4}}=0.572,\left(v_{B}\right)_{k_{5}}=0.004,\left(v_{B}\right)_{k_{6}}=0.124$.

\section{Список литературы}

[1] L. Ci, L. Song, C. Jin, D. Jariwala, D. Wu, Y. Li, A. Srivastava, Z.F. Wang, K. Storr, L. Balicas, F. Liu, P.M. Ajayan. Nature Mater. 9, 430 (2010).

[2] M.P. Levendorf, C.-J. Kim, L. Brown, P.Y. Huang, R.W. Havener, D.A. Muller, J. Park. Nature 488, 627 (2012).

[3] Z. Liu, L. Ma, G. Shi, W. Zhou, Y. Gong, S. Lei, X. Yang, J. Zhang, J. Yu, K.P. Hackenberg, A. Babakhani, J.-C. Idrobo, R. Vajtai, J. Lou, P.M. Ajayan. Nature Nanotechnology 8, 119 (2013).

[4] G.C. Loh, R. Pandey. J. Mater. Chem. C 3, 5918 (2015).

[5] J. Zhang, W. Xie, X. Xu, S. Zhang, J. Zhao. Chem. Mater 28, 5022 (2016).

[6] Z.-Y. Ong, G. Zhang, Y.-W. Zhang. Phys. Rev. B 93, 075406 (2016).

[7] И.В. Антонова. ФТП 50, 67 (2016).

[8] С.Ю. Давыдов. Простые модели латеральных гетероструктур. ФТТ 60, 1815 (2018).

[9] С.Ю. Давыдов. ФТТ 59, 1650 (2017).

[10] С.Ю. Давыдов, А.А. Лебедев, О.В. Посредник. Элементарное введение в теорию наносистем. Лань, СПб. (2014). $192 \mathrm{c}$.

[11] С.Ю. Давыдов. Теория адсорбции: метод модельных гамильтонианов. СПб.: Изд-во СПбГЭТУ ЛЭТИ, СПб. (2013). 235 c. twirpx.com/file/1596114/ 
[12] С.Ю. Давыдов. ФТТ 54, 2193 (2012).

[13] У. Харрисон. Электронная структура и свойства твердых тел. Мир, М. (1983). Т. 1, 382 с.

[14] W.A. Harrison. Phys. Rev. B 27, 3592 (1983).

[15] С.Ю. Давыдов. ФТП 47, 97 (2013).

[16] С.Ю. Давыдов. ФТТ 58, 779 (2016).

[17] O. Leenaerts, B. Partoens, F.M. Peeters. Phys. Rev. B 77. 125416 (2008)

[18] С.Ю. Давыдов, О.В. Посредник. ФТТ 57, 819 (2015).

[19] В.Ю. Ирхин, Ю.П. Ирхин. Электронная структура, физические свойства и корреляционные эффекты в $d$ - и $f$-металлах и их соединениях. УрО РАН, Екатеринбург (2004). $472 \mathrm{c}$.

[20] С.Ю. Давыдов. ФТП 52, 240 (2018).

Редактор Т.Н. Василевская 